\title{
Biosynthesis of LC-PUFA and VLC-PUFA in Pampus argenteus: Characterization of Elovl4 elongases and regulation under acute salinity
}

Jiaxiang Luo ${ }^{1 \#}$, Óscar Monroig ${ }^{2 *}$ Kai Liao $^{3}$, Alberto Ribes-Navarro², Juan Carlos Navarro², Tingting Zhu' ${ }^{1}$, Juan $\mathrm{Li}^{3}$, Liangli Xue ${ }^{3}$, Qicun Zhou ${ }^{1 *}$, Min Jin ${ }^{1 *}$

\author{
Affiliations \\ ${ }^{1}$ Laboratory of Fish and Shellfish Nutrition, School of Marine Sciences, Ningbo University, Ningbo, \\ China \\ ${ }^{2}$ Instituto de Acuicultura Torre de la Sal (IATS-CSIC), 12595 Ribera de Cabanes, Castellón, Spain \\ ${ }^{3}$ School of Marine Sciences, Ningbo University, Ningbo, China
}

\section{Corresponding authors}

Dr. / Prof. Qicun Zhou, Tel/Fax: +86 574 87609878, zhouqicun@nbu.edu.cn (Q. Zhou).

Dr. Óscar Monroig, Tel: +34 964 319500, oscar.monroig@csic.es (Ó. Monroig).

Dr. Min Jin, Tel/Fax: +86 574 87609878, jinmin@nbu.edu.cn (M. Jin). 


\section{Contents:}

Table S1. Primers used in this study

Table S2. Fatty acid compositions (mg/g dry matter) of experimental diet

Table S3. Fatty acid compositions (mg/g dry matter) of $P$. argenteus in liver cultured in different salinity .

Table S4. Fatty acid compositions (mg/g dry matter) of $P$. argenteus in gill cultured in different salinity. 
Table S1. Primers used in this study

\begin{tabular}{|c|c|c|}
\hline Aim & Primer name & Primer sequence (5'-3') \\
\hline \multirow{4}{*}{$\begin{array}{l}\text { Partial fragment cDNA } \\
\text { cloning }\end{array}$} & Elovl4a-F & TTGCAGACAAGCGGGTGGA \\
\hline & Elov14a-R & CCGAAGAGGATGATGAAGGTGA \\
\hline & Elovl4b-F & TTGTGATGGGCTTGCCTCCCTTAT \\
\hline & Elovl4b-R & ACCCTCATAGTCTACAACTTCAGC \\
\hline \multirow[t]{6}{*}{ 3' RACE PCR } & Elovl4a-3R-F1 & CATCTGCTACGCCATCACCTT \\
\hline & Elov14a-3R-F2 & GCAGGAGGGCAGTCATTCTTT \\
\hline & Elovl4a-3R-F3 & GGACTATTCGGACGACCCC \\
\hline & Elovl4b-3R-F1 & ATGATGTCAATGAAGTCAGGATAGCA \\
\hline & Elovl4b-3R-F2 & CACGTCCTCATGTACGGTTACTATGG \\
\hline & Elovl4b-3R-F3 & ACCACGCTTACCGACGTAAACCTTCC \\
\hline \multirow[t]{6}{*}{ 5' RACE PCR } & Elovl4a-5R-R1 & AGGAACAGCAGGTAGGAGGTG \\
\hline & Elov14a-5R-R2 & AGTCCACTGGTTGGCAAATGT \\
\hline & Elovl4a-5R-R3 & TTGATGCCAATCCACCAGAGC \\
\hline & Elovl4b-5R-R1 & AGGTGACAGCATAGCCAATCAGAGC \\
\hline & Elov14b-5R-R2 & GCACCAAAAAAAGATTGTCCACCTG \\
\hline & Elov14b-5R-R3 & GCAGATGCTATCCTGACTTCATTGA \\
\hline \multirow[t]{6}{*}{$\begin{array}{l}\text { ORF cloning for functional } \\
\text { characterization }\end{array}$} & Elovl4a-V-F & $\begin{array}{l}\text { CCCAAGCTTATGGAGATTGTAACACAT } \\
\text { TTAAT }\end{array}$ \\
\hline & Elovl4a-V-R & $\begin{array}{l}\text { CCCСTCGAGCTAATCTCTTTTGGCTCTT } \\
\text { ССТTT }\end{array}$ \\
\hline & Elovl4b-V-F & $\begin{array}{l}\text { CCCAAGCTTATGGAGGCTGTAACACAT } \\
\text { CTGG }\end{array}$ \\
\hline & Elovl4b-V-R & $\begin{array}{l}\text { CCCGAATTCTTACTCCCTTTTCGCTCTT } \\
\text { CC }\end{array}$ \\
\hline & Fads2-V-F & $\begin{array}{l}\text { CGGGGTACCATGGGTGGTGGAGGCCA } \\
\text { ACT }\end{array}$ \\
\hline & Fads2-V-R & $\begin{array}{l}\text { CCGCTCGAGTCATTTATGAAGATATGC } \\
\text { AT }\end{array}$ \\
\hline \multirow[t]{11}{*}{ qPCR } & Elovl4a-Q-F & ACTTCTACTACCAGACCTACCG \\
\hline & Elovl4a-Q-R & TTTTGGCTCTTCCTTTCC \\
\hline & Elovl4b-Q-F & TTGGCTATGCTGTCACCT \\
\hline & Elovl4b-Q-R & TTACTCCCTTTTCGCTCT \\
\hline & $18 S-\mathrm{F}$ & AGTTGGTGGAGCGATTTGTCTGGTTA \\
\hline & $18 S-\mathrm{R}$ & CTAAGAAGTTGGACGCCGACCGCACG \\
\hline & Elovl5-F & GGGGATTTCTGCTGCTGGAC \\
\hline & Elovl5-R & AGATTGTAGAGCACCAGGAGGC \\
\hline & Fads2-F & ATGCTGACGCTGCTGTGCTC \\
\hline & Fads2-R & ATGCTGGAAATGTCGATGGTT \\
\hline & Srebp-F & CAGGACATGGAGTTTGGAGG \\
\hline
\end{tabular}




\begin{tabular}{ll} 
Srebp- $R$ & AGGATGGAGGAGGACTTGGAT \\
Lxr $-F$ & TCTGCAGGGAGAACCGAATG \\
Lxr $\alpha-R$ & CGCTGCACACTTCATTACCC \\
Ppar $\alpha-F$ & CCACTACCAACCCCCTTCAC \\
Ppar $-R$ & GGAGCTAAGGACGCTGTTGT \\
$H n f 4 \alpha-F$ & GTGGACAAAGACAAGCGAAAT \\
$H n f 4 \alpha-R$ & GTTCTTATGTCGCCGTTCAG \\
\hline
\end{tabular}


Table S2. Fatty acid compositions (mg/g dry matter) of experimental diet

\begin{tabular}{|c|c|}
\hline Parameter & Diet \\
\hline $14: 0$ & 2.79 \\
\hline $16: 0$ & 18.82 \\
\hline $18: 0$ & 5.29 \\
\hline $20: 0$ & 0.24 \\
\hline$\Sigma \mathrm{SFA}^{a}$ & 27.15 \\
\hline $16: 1 n$ & 4.41 \\
\hline $18: 1 n-9$ & 12.25 \\
\hline $20: 1 n-9$ & 0.59 \\
\hline $22: 1 n-11$ & 0.06 \\
\hline$\Sigma$ MUFA $^{b}$ & 17.32 \\
\hline $18: 2 n-6$ & 3.33 \\
\hline $18: 3 n-6$ & 0.39 \\
\hline $20: 2 n-6$ & 0.11 \\
\hline $20: 4 n-6$ & 0.34 \\
\hline $22: 4 n-6$ & 0.09 \\
\hline$\Sigma$ n-6 PUFA ${ }^{c}$ & 4.27 \\
\hline $18: 3 n-3$ & 0.53 \\
\hline $18: 4 n-3$ & 0.29 \\
\hline $20: 4 n-3$ & 0.28 \\
\hline $20: 5 n-3$ & 3.82 \\
\hline $22: 5 n-3$ & 0.80 \\
\hline $22: 6 n-3$ & 8.43 \\
\hline$\Sigma \mathrm{n}-3 \mathrm{PUFA}^{d}$ & 14.16 \\
\hline n-3/n-6 PUFA ${ }^{e}$ & 3.31 \\
\hline$\Sigma \mathrm{n}-3$ LC-PUFA ${ }^{f}$ & 13.34 \\
\hline$\Sigma$ n-6 LC-PUFA ${ }^{g}$ & 0.55 \\
\hline$\Sigma$ LC-PUFA $^{\mathrm{h}}$ & 13.88 \\
\hline
\end{tabular}

Some fatty acids, of which the contents are minor, trace amount or not detected, such as 22:0, 24:0, 14:1n-7, 22:1n-9, 20:3n-6, 20:5n-6, 20:3n-3 were not listed in Table S2.

${ }^{a}$ SFA, saturated fatty acids.

${ }^{b}$ MUFA, mono-unsaturated fatty acids.

${ }^{c}$ n-6 PUFA, n-6 polyunsaturated fatty acids.

${ }^{d}$ n-3 PUFA, n-3 polyunsaturated fatty acids.

${ }^{e}$ n-3/n-6 PUFA, n-3 polyunsaturated fatty acids: n-6 polyunsaturated fatty acids. 
Table S3. Fatty acid compositions (mg/g dry matter) of $P$. argenteus in liver cultured in different salinity .

\begin{tabular}{|c|c|c|c|}
\hline \multirow{2}{*}{ Parameter } & \multicolumn{3}{|l|}{ Salinity } \\
\hline & $12 \mathrm{ppt}$ & $22 \mathrm{ppt}$ & $32 \mathrm{ppt}$ \\
\hline $14: 0$ & $3.31 \pm 0.30$ & $4.05 \pm 3.09$ & $3.09 \pm 0.17$ \\
\hline $16: 0$ & $27.53 \pm 0.68^{\mathrm{ab}}$ & $28.32 \pm 0.77^{\mathrm{a}}$ & $24.85 \pm 0.12^{\mathrm{b}}$ \\
\hline $18: 0$ & $7.37 \pm 0.01^{\mathrm{a}}$ & $6.55 \pm 0.50^{\mathrm{b}}$ & $6.38 \pm 0.08^{\mathrm{b}}$ \\
\hline $20: 0$ & $0.21 \pm 0.04$ & $0.20 \pm 0.01$ & $0.16 \pm 0.01$ \\
\hline$\Sigma \mathrm{SFA}^{a}$ & $38.41 \pm 0.33^{\mathrm{a}}$ & $39.13 \pm 0.67^{\mathrm{a}}$ & $34.49 \pm 0.13^{\mathrm{b}}$ \\
\hline $16: 1 n$ & $6.90 \pm 0.79$ & $8.89 \pm 0.05$ & $6.70 \pm 0.60$ \\
\hline $18: 1 n-9$ & $48.31 \pm 3.43$ & $44.16 \pm 5.90$ & $34.63 \pm 4.72$ \\
\hline $20: 1 n-9$ & $1.72 \pm 0.16$ & $1.46 \pm 0.24$ & $1.02 \pm 0.08$ \\
\hline $22: 1 n-11$ & $0.65 \pm 0.05^{\mathrm{a}}$ & $0.44 \pm 0.01^{\mathrm{b}}$ & $0.49 \pm 0.06^{\mathrm{ab}}$ \\
\hline$\Sigma \mathrm{MUFA}^{b}$ & $57.58 \pm 4.09$ & $54.95 \pm 6.09$ & $42.85 \pm 5.45$ \\
\hline $18: 2 n-6$ & $1.59 \pm 0.18$ & $1.85 \pm 0.04$ & $1.80 \pm 0.09$ \\
\hline $18: 3 n-6$ & $0.13 \pm 0.01$ & $0.13 \pm 0.01$ & $0.11 \pm 0.01$ \\
\hline $20: 2 n-6$ & $0.19 \pm 0.02$ & $0.20 \pm 0.02$ & $0.18 \pm 0.00$ \\
\hline $20: 4 n-6$ & $2.48 \pm 0.08$ & $2.46 \pm 0.10$ & $2.33 \pm 0.05$ \\
\hline $22: 4 n-6$ & $0.4 \pm 0.03^{\mathrm{a}}$ & $0.28 \pm 0.02^{\mathrm{b}}$ & $0.19 \pm 0.01^{\mathrm{c}}$ \\
\hline$\Sigma \mathrm{n}-6 \mathrm{PUFA}^{c}$ & $4.79 \pm 0.15$ & $4.92 \pm 0.11$ & $4.62 \pm 0.07$ \\
\hline $18: 3 n-3$ & $0.35 \pm 0.04$ & $0.44 \pm 0.05$ & $0.34 \pm 0.04$ \\
\hline $18: 4 n-3$ & $0.35 \pm 0.02$ & $0.41 \pm 0.08$ & $0.35 \pm 0.08$ \\
\hline $20: 4 n-3$ & $0.93 \pm 0.02$ & $0.93 \pm 0.09$ & $0.75 \pm 0.05$ \\
\hline $20: 5 n-3$ & $6.12 \pm 0.09$ & $6.77 \pm 0.62$ & $6.13 \pm 0.07$ \\
\hline $22: 5 n-3$ & $6.22 \pm 0.01^{\mathrm{a}}$ & $4.94 \pm 0.63^{\mathrm{ab}}$ & $4.32 \pm 0.34^{\mathrm{b}}$ \\
\hline $22: 6 n-3$ & $30.22 \pm 0.98^{\mathrm{a}}$ & $29.03 \pm 1.03^{\mathrm{a}}$ & $26.72 \pm 0.29^{b}$ \\
\hline$\Sigma$ n-3 PUFA $^{d}$ & $44.20 \pm 1.01^{\mathrm{a}}$ & $42.52 \pm 1.70^{\mathrm{ab}}$ & $38.60 \pm 0.54^{b}$ \\
\hline n-3/n-6 PUFA ${ }^{e}$ & $9.25 \pm 0.49$ & $8.66 \pm 0.51$ & $8.37 \pm 0.17$ \\
\hline$\Sigma$ n-3 LC-PUFA ${ }^{f}$ & $43.50 \pm 1.06^{\mathrm{a}}$ & $41.67 \pm 1.60^{\mathrm{ab}}$ & $37.91 \pm 0.64^{\mathrm{b}}$ \\
\hline$\Sigma$ n-6 LC-PUFA ${ }^{g}$ & $3.07 \pm 0.05^{\mathrm{a}}$ & $2.94 \pm 0.07^{\mathrm{a}}$ & $2.70 \pm 0.04^{\mathrm{b}}$ \\
\hline$\Sigma$ LC-PUFA $^{\mathrm{h}}$ & $46.57 \pm 1.10^{\mathrm{a}}$ & $44.62 \pm 1.54^{\mathrm{ab}}$ & $40.61 \pm 0.67^{\mathrm{b}}$ \\
\hline
\end{tabular}

Data represent mean \pm S.E.M. of three replicates $(n=3)$. Values in the same column with different superscripts are significantly different $(P<0.05)$. Some fatty acids, of which the contents are minor, trace amount or not detected, such as 22:0, 24:0, 14:1n-7, 22:1n-9, 20:3n-6, 20:5n-6, 20:3n-3 were not listed in Table S2.

${ }^{a}$ SFA, saturated fatty acids.

${ }^{b}$ MUFA, mono-unsaturated fatty acids.

${ }^{c} \mathrm{n}-6$ PUFA, $\mathrm{n}-6$ polyunsaturated fatty acids.

${ }^{d} \mathrm{n}-3$ PUFA, n-3 polyunsaturated fatty acids.

${ }^{e}$ n-3/n-6 PUFA, n-3 polyunsaturated fatty acids: n-6 polyunsaturated fatty acids. 
${ }^{f}$ n-3 LC-PUFA, n-3 long-chain polyunsaturated fatty acids.

${ }^{g}$ n-6 LC-PUFA, n-6 long-chain polyunsaturated fatty acids.

${ }^{h}$ LC-PUFA, long-chain $\left(\mathrm{C}_{20-24}\right)$ polyunsaturated fatty acids. 
Table S4. Fatty acid compositions (mg/g dry matter) of $P$. argenteus in gill cultured in different salinity.

\begin{tabular}{|c|c|c|c|}
\hline \multirow{2}{*}{ Parameter } & \multicolumn{3}{|l|}{ Salinity } \\
\hline & $12 \mathrm{ppt}$ & $22 \mathrm{ppt}$ & $32 \mathrm{ppt}$ \\
\hline $14: 0$ & $6.42 \pm 0.62$ & $7.65 \pm 0.55$ & $7.24 \pm 0.45$ \\
\hline $16: 0$ & $25.31 \pm 2.30$ & $26.61 \pm 1.99$ & $27.32 \pm 0.61$ \\
\hline 18:0 & $6.46 \pm 0.49$ & $5.93 \pm 0.40$ & $6.42 \pm 0.36$ \\
\hline $20: 0$ & $0.55 \pm 0.02$ & $0.59 \pm 0.06$ & $0.49 \pm 0.02$ \\
\hline$\Sigma \mathrm{SFA}^{a}$ & $38.73 \pm 3.38$ & $40.78 \pm 2.92$ & $41.47 \pm 1.24$ \\
\hline $16: 1 \mathrm{n}$ & $8.68 \pm 0.95$ & $10.3 \pm 1.09$ & $9.74 \pm 0.58$ \\
\hline $18: 1 n-9$ & $42.52 \pm 4.08$ & $46.71 \pm 5.61$ & $46.81 \pm 1.9$ \\
\hline $20: 1 n-9$ & $1.96 \pm 0.25$ & $2.27 \pm 0.28$ & $1.94 \pm 0.13$ \\
\hline $22: 1 n-11$ & $0.88 \pm 0.14$ & $0.83 \pm 0.02$ & $0.91 \pm 0.05$ \\
\hline$\Sigma \mathrm{MUFA}^{b}$ & $54.04 \pm 5.28$ & $60.11 \pm 6.94$ & $59.41 \pm 2.55$ \\
\hline $18: 2 n-6$ & $2.58 \pm 0.26$ & $3.23 \pm 0.53$ & $3.47 \pm 0.28$ \\
\hline $18: 3 n-6$ & $0.13 \pm 0.01^{\mathrm{a}}$ & $0.22 \pm 0.02^{\mathrm{b}}$ & $0.17 \pm 0.02^{\mathrm{ab}}$ \\
\hline $20: 2 n-6$ & $0.17 \pm 0.02$ & $0.24 \pm 0.04$ & $0.2 \pm 0.01$ \\
\hline $20: 4 n-6$ & $1.82 \pm 0.04$ & $1.80 \pm 0.1$ & $1.95 \pm 0.07$ \\
\hline $22: 4 n-6$ & $0.46 \pm 0.03$ & $0.61 \pm 0.11$ & $0.60 \pm 0.01$ \\
\hline$\Sigma$ n-6 PUFA ${ }^{c}$ & $5.16 \pm 0.31$ & $6.09 \pm 0.79$ & $6.39 \pm 0.30$ \\
\hline $18: 3 n-3$ & $0.68 \pm 0.08$ & $0.85 \pm 0.12$ & $0.80 \pm 0.07$ \\
\hline $18: 4 n-3$ & $1.20 \pm 0.17$ & $1.43 \pm 0.14$ & $1.25 \pm 0.09$ \\
\hline $20: 4 n-3$ & $1.31 \pm 0.14$ & $1.37 \pm 0.08$ & $1.43 \pm 0.05$ \\
\hline $20: 5 n-3$ & $7.54 \pm 0.58$ & $7.53 \pm 0.66$ & $7.46 \pm 0.41$ \\
\hline $22: 5 n-3$ & $4.60 \pm 0.36$ & $4.97 \pm 0.59$ & $4.66 \pm 0.34$ \\
\hline $22: 6 n-3$ & $16.34 \pm 0.92$ & $16.52 \pm 1.30$ & $15.23 \pm 0.60$ \\
\hline$\Sigma$ n-3 PUFA $^{d}$ & $31.67 \pm 2.23$ & $32.68 \pm 2.74$ & $30.93 \pm 1.47$ \\
\hline n-3/n-6 PUFA ${ }^{e}$ & $6.13 \pm 0.15^{\mathrm{a}}$ & $5.44 \pm 0.29^{\mathrm{ab}}$ & $4.85 \pm 0.15^{\mathrm{b}}$ \\
\hline$\Sigma$ n-3 LC-PUFA ${ }^{f}$ & $29.80 \pm 1.99$ & $30.39 \pm 2.48$ & $28.88 \pm 1.38$ \\
\hline$\Sigma$ n-6 LC-PUFA ${ }^{g}$ & $2.45 \pm 0.06$ & $2.64 \pm 0.25$ & $2.76 \pm 0.05$ \\
\hline$\Sigma$ LC-PUFA ${ }^{h}$ & $35.64 \pm 2.34$ & $36.73 \pm 3.02$ & $34.98 \pm 1.54$ \\
\hline
\end{tabular}

Data represent mean \pm S.E.M. of three replicates $(n=3)$. Values in the same column with different superscripts are significantly different $(P<0.05)$. Some fatty acids, of which the contents are minor, trace amount or not detected, such as 22:0, 24:0, 14:1n-7, 22:1n-9, 20:3n-6, 20:5n-6, 20:3n-3 were not listed in Table S3.

${ }^{a}$ SFA, saturated fatty acids.

${ }^{b}$ MUFA, mono-unsaturated fatty acids.

${ }^{c} \mathrm{n}-6$ PUFA, $\mathrm{n}-6$ polyunsaturated fatty acids.

${ }^{d}$ n-3 PUFA, n-3 polyunsaturated fatty acids.

${ }^{e}$ n-3/n-6 PUFA, n-3 polyunsaturated fatty acids: n-6 polyunsaturated fatty acids. 
${ }^{f}$ n-3 LC-PUFA, n-3 long-chain polyunsaturated fatty acids.

${ }^{g}$ n-6 LC-PUFA, n-6 long-chain polyunsaturated fatty acids.

${ }^{h}$ LC-PUFA, long-chain $\left(\mathrm{C}_{20-24}\right)$ polyunsaturated fatty. 